> Les protéines vitamine K-dépendantes sont essentiellement connues pour leur implication dans la coagulation du sang. Récemment, deux protéines vitamine K-dépendantes, le facteur anticoagulant protéine $S$ et son homologue structural Gas-6, ont été identifiés comme ligands des récepteurs à activité tyrosine kinase TAM (Tyro-3/Axl/Mer). L'analyse des phénotypes de souris transgéniques invalidées pour les gènes codant pour Gas- 6 ou pour ses récepteurs a révélé que Gas-6 et la protéine $S$ sont impliquées dans la régulation de la phagocytose des cellules apoptotiques, processus important dans la réponse immunitaire ainsi que dans les processus de différenciation cellulaire. Les nouvelles fonctions de ces protéines vitamine K-dépendantes ainsi que leur utilisation potentielle dans l'élaboration de traitements de pathologies associées à un déficit de phagocytose font l'objet de cet article. <

\section{La $\boldsymbol{\gamma}$-carboxylation :} une modification post-traductionnelle impliquant la $\gamma$-carboxylase et le complexe vitamine $K$ réductase

La vitamine $K$ ( $K$ pour Koagulation) a été identifiée par les lauréats du prix Nobel de médecine et physiologie en 1943, Karl Dam et Edwards Doisy, comme nutriment essentiel à la coagulation du sang. La coagulation sanguine est régulée par une succession de clivages protéolytiques de facteurs plasmatiques sécrétés par le foie dont certains ne peuvent être produits sous forme active qu'en présence de vitamine $K$ [1]. De ce fait, un déficit en vitamine $K$ conduit à des troubles de la coagulation sanguine.

La vitamine $K$ agit en tant que cofacteur de l'enzyme $\gamma$-glutamyl carboxylase qui catalyse une modification post-traductionelle, la $\gamma$-carboxylation. Cette réaction consiste en la substitution, au niveau du carbone $\gamma$ d'un acide glutamique, d'un atome d'hydrogène par un groupement carboxyle, le modifiant ainsi en acide

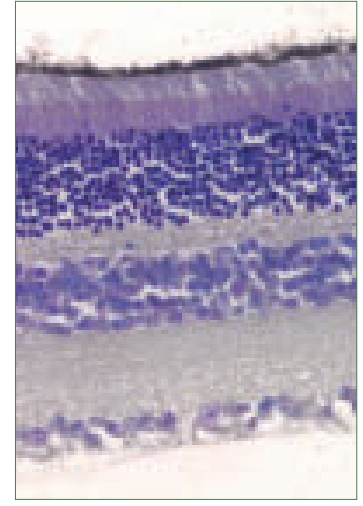

Institut de Physiologie et Biologie Cellulaires; CNRS-UMR 6187 ; Pôle Biologie Santé, Université de Poitiers, 40, avenue du Recteur Pineau, 86022 Poitiers Cedex, France. 0mar.Benzakour@ $\underline{\text { univ-poitiers.fr }}$

$\gamma$-carboxyglutamique (résidu Gla) [2] (Figure 1). La $\gamma$-glutamyl carboxylase nécessite comme cofacteur la vitamine $\mathrm{K}$ sous sa forme réduite (hydroquinone) et engendre du $\gamma$-carboxyglutamate et de la vitamine $K$ sous sa forme oxydée (2,3,-époxyde). La vitamine $K 2,3$,-époxyde réductase ou VKOR recycle la vitamine $K$ oxydée permettant ainsi au cycle de la $\gamma$-carboxylation de continuer [2]. Les protéines $\gamma$-carboxylées sont caractérisées par un domaine consistant en une répétition d'acides $\gamma$-carboxyglutamiques également appelé «domaine-Gla». Ce domaine contient un site consensus de fixation pour la $\gamma$-carboxylase dont la séquence est : $Z-F-X-X-X-X-A$ où $Z$ correspond à un acide aminé hydrophobe aliphatique, $F$ à une phénylalanine, $X$ à un acide aminé quelconque et $A$ à l'alanine [3]. La $\gamma$-carboxylation est nécessaire à l'activité biologique des protéines $\gamma$-carboxylées, d'où leur dénomination de protéines vitamine K-dépendantes $[2,3]$. Il est admis que le domaine Gla confère des charges négatives qui permettent à ces protéines de lier le calcium et d'interagir avec les phospholipides membranaires [2, 3].

La Warfarine (3-[ $\alpha$-acetonyl-benzyl-4-hydroxycoumarine]), dont le nom renvoie à I'Université du Winsconsin où elle a été découverte et qui en possède les droits (Wisconsin Alumni Research Foundation), est un antagoniste de la vitamine K. Elle 
bloque le recyclage de la vitamine $\mathrm{K}$ oxydée et inhibe ainsi le cycle de la $\gamma$-carboxylation [2]. C'est en vertu de cette propriété que la Warfarine et ses dérivés sont utilisés comme traitements anticoagulants, connus aussi sous le terme de traitements AVK (anti-vitamine K) (Figure l). En l'an 2000, la Warfarine était classée onzième au palmarès des médicaments les plus vendus aux États-Unis avec un chiffre d'affaires estimé à 500 millions de dollars [4].

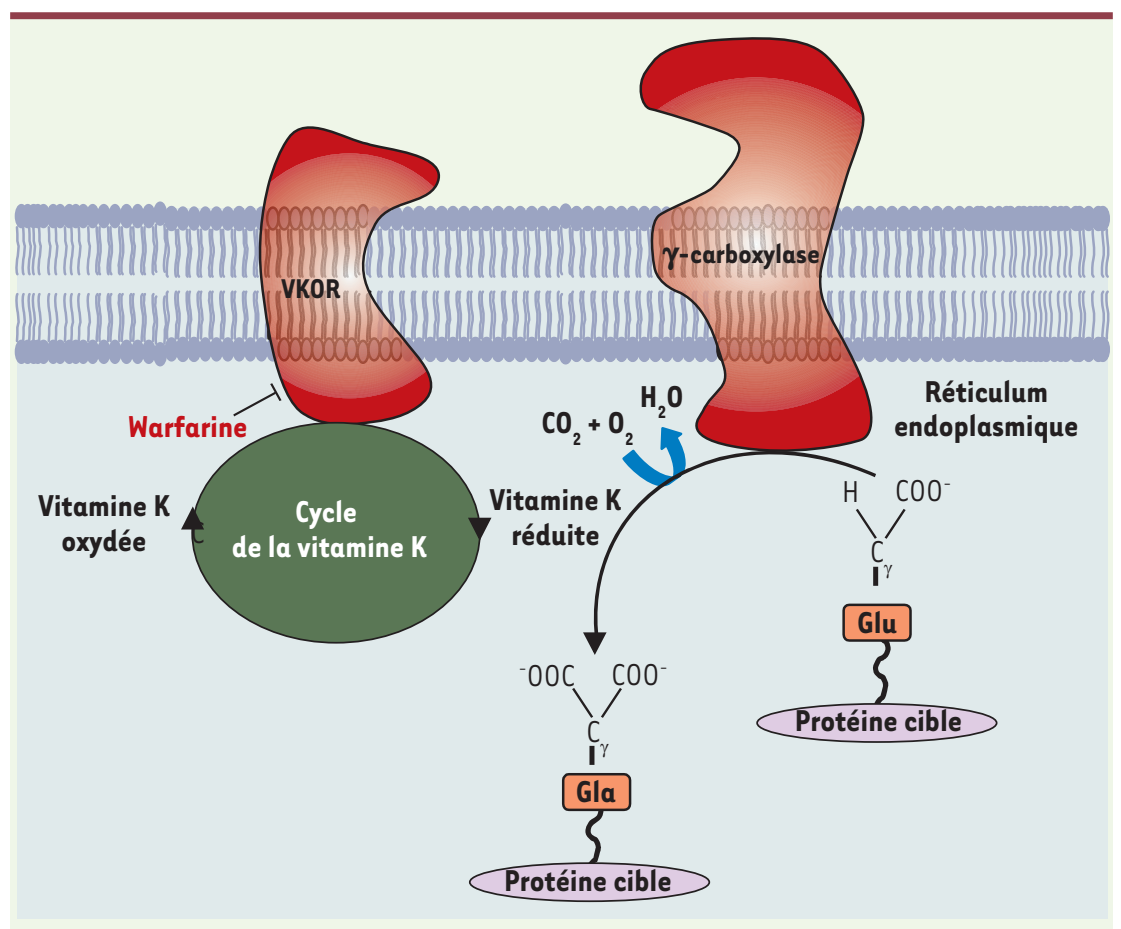

Figure 1. Couplage entre la réaction de $\gamma$-carboxylation et le cycle de la vitamine $K$ au niveau du réticulum endoplasmique. La $\gamma$-carboxylation du glutamate (Glu) en $\gamma$-carboxyglutamate (Gla) de précurseurs protéiques est couplée à l'oxydation de la Vitamine K1. La vitamine K 2,3,époxyde réductase ou VKOR recycle la vitamine K oxydée. La Warfarine inhibe le recyclage de la vitamine Kl réduite par l'époxyde réductase (VKOR).

\section{La $\gamma$-carboxylation ne concerne qu'un nombre restreint de protéines}

Les protéines vitamine K-dépendantes constituent une famille de 14 membres qui peuvent être répartis, selon leurs fonctions connues ou supposées, en quatre catégories majeures: (1) plusieurs protéines du système de la coagulation sanguine dont la prothrombine (ou facteur II), les facteurs VII, IX, X, la protéine $Z$, la protéine $C$, la protéine $S$; (2) une protéine codée par le gène GAS6 (growth arrest specific gene 6) découvert par criblage différentiel de banques d'ADNc de cellules NIH3T3 en état d'arrêt de croissance par rapport aux cellules en croissance exponentielle, le suffixe 6 se réfèrant au numéro du clone; (3) deux protéines intervenant dans la calcification, l'ostéocalcine et la Gla-protéine de la matrice (matrix Gla protein ou MGP) ; et (4) quatre Gla-protéines transmembranaires (PRGP1, PRGP2, TMG3 et TMG4) dont la fonction est jusqu'à présent inconnue (Tableau / et II).

Hormis la protéine S, Gas6 et la protéine $Z$, les protéines vitamine $K$-dépendantes de la cascade de la coagulation du sang sont à la fois des sérines protéases et sont activables par des sérines protéases (Tableau 1) [5, 6]. Le rôle de la protéine $Z$ dans la coagulation du sang n'est pas entièrement élucidé et aucun effet cellulaire ne lui a été attribué. La thrombine, mais aussi le facteur $\mathrm{Xa}$, le facteur VIla et la protéine $C$ activée, activent des mem-

\begin{tabular}{|c|c|c|c|c|}
\hline Facteur & $\begin{array}{l}\text { Lieu } \\
\text { de synthèse }\end{array}$ & Fonction dans la coagulation du sang & Récepteurs cellulaires & Références \\
\hline $\begin{array}{l}\text { Prothrombine } \\
\text { ou facteur II }\end{array}$ & Foie & $\begin{array}{l}\text { Zymogène du Flla procoagulant, la thrombine } \\
\text { active le FVIII }\end{array}$ & $\mathrm{PAR}_{1} \mathrm{PAR}_{3} \mathrm{PAR}_{4}$ & {$[5,7]$} \\
\hline Facteur VII & Foie & $\begin{array}{l}\text { Zymogène du FVIla procoagulant activé par Flla. } \\
\text { Active les FIX et X. }\end{array}$ & $\mathrm{PAR}_{2}$ & {$[6,36]$} \\
\hline Facteur IX & Foie & $\begin{array}{l}\text { Zymogène du FVIla procoagulant activé par le Flla. } \\
\text { Active les FIX et X. }\end{array}$ & Inconnu & [37] \\
\hline Facteur X & Foie & $\begin{array}{l}\text { Zymogène du FXa procoagulant activé par FVIla et } \\
\text { IXa. Active le facteur II. }\end{array}$ & $\mathrm{PAR}_{1} \mathrm{PAR}_{2}$ & [36 ] \\
\hline Protéine C & Foie & $\begin{array}{l}\text { Zymogène de la protéine C activée anticoagulante. } \\
\text { Inactive les FVa et VIIla. }\end{array}$ & $\varepsilon P C R, P_{1}, P_{1} R_{3}$ & {$[38,39]$} \\
\hline
\end{tabular}

Tableau I. Protéines vitamine K-dépendantes avec activité protéolytique. 


\begin{tabular}{|c|c|c|c|}
\hline Protéine & Lieu de synthèse & Fonctions et effets cellulaires & Références \\
\hline Protéine Z & Foie & $\begin{array}{l}\text { Absence d'effets cellulaires, cofacteur } \\
\text { dans l'inhibition du FXa }\end{array}$ & {$[40]$} \\
\hline Protéine S & $\begin{array}{l}\text { Foie, cerveau, cœur, ovaire, placenta, rate, rein, } \\
\text { cellules endothéliales, mégacaryocytes, } \\
\text { ostéoblastes, cellules du système nerveux, } \\
\text { cellules vasculaires lisses et cellules de Leydig }\end{array}$ & $\begin{array}{l}\text { Cofacteur de la protéine C. Stimule la résorption } \\
\text { osseuse. Participe à la phagocytose des cellules } \\
\text { apoptotiques et au shedding des photorécepteurs } \\
\text { rétiniens }\end{array}$ & $\begin{array}{c}{[8,19,30,41,} \\
42]\end{array}$ \\
\hline Gas6 & $\begin{array}{l}\text { Cœur, cerveau, poumons, estomac, rein, intestin, } \\
\text { rétine, testicules (cellules de Leydig et cellules } \\
\text { de Sertoli), pancréas, os (ostéoclastes } \\
\text { et ostéoblastes) et cellules endothéliales }\end{array}$ & $\begin{array}{l}\text { Stimule la survie, la prolifération, la migration, } \\
\text { l'adhésion cellulaires ainsi que la résorption } \\
\text { osseuse. Participe à la reconnaissance et à la } \\
\text { phagocytose des cellules apoptotiques et au } \\
\text { shedding des photorécepteurs rétiniens }\end{array}$ & {$[8,19,31,41]$} \\
\hline MGP & $\begin{array}{l}\text { Cellules vasculaires du muscle lisse } \\
\text { et chondrocytes }\end{array}$ & $\begin{array}{l}\text { Inhibiteur de la calcification de la matrice } \\
\text { extracellulaire }\end{array}$ & [43] \\
\hline $\begin{array}{l}\text { BGP/ } \\
\text { Ostéocalcine }\end{array}$ & Ostéoblastes et odontoblastes & Inhibiteur de la formation osseuse & {$[44]$} \\
\hline $\begin{array}{l}\text { TMG3 } \\
\text { TMG4 }\end{array}$ & $\begin{array}{l}\text { ARNm : cerveau et cœur pour TMG3 et rein } \\
\text { et pancréas pour TMG } 4\end{array}$ & inconnu & {$[45]$} \\
\hline $\begin{array}{l}\text { PRGP1 } \\
\text { PRGP2 }\end{array}$ & $\begin{array}{l}\text { ARNm : placenta et pancréas pour PRGP1 } \\
\text { et PRGP2 et rein pour PRGP2 }\end{array}$ & inconnu & {$[46]$} \\
\hline
\end{tabular}

Tableau II. Protéines vitamine K-dépendantes sans activité protéolytique.

bres de la famille des récepteurs couplés aux protéines $\mathrm{G}$ activables par protéolyse, les PAR. II existe aujourd'hui une littérature abondante sur ce domaine [5-7]. Aussi allons nous focaliser la suite de cet article sur la protéine $S$ et la protéine Gas6, qui présentent les particularités d'être sécrétées par un grand nombre de types cellulaires, d'activer une classe particulière de récepteurs à activité tyrosine kinase et d'avoir récemment fait l'objet d'importants travaux de recherche pour leur implication dans une grande diversité de processus physiopathologiques.

\section{La protéine S et la protéine Gas6: de vrais fausses jumelles}

La protéine S (S pour Seattle) et son homologue structural Gas6 (growth arrest-specific gene 6) sont des glycoprotéines sécrétées de 69 et $75 \mathrm{kDa}$. La protéine Gas 6 possède $44 \%$ d'homologie de séquence avec la protéine $S$ et les domaines structuraux de ces deux protéines sont presque identiques. En effet, à part le site de clivage putatif par des sérines protéases, propre à la protéine $S$, Gas6 et la protéine $S$ présentent un domaine Gla amino-terminal, une répétition de quatre domaines EGF (epidermal growth factor)-like et un domaine SHBG (sex hormone-binding globulin) [8] (Figure 2A). C'est par leur domaine SHBG que Gas6 et la protéine $S$ interagissent avec leurs récepteurs membranaires (Figure 2b). À la différence des autres protéines du système de la coagulation du sang dont l'expression est limitée au foie, la protéine $S$ et la protéine Gas6 sont produites par une grande variété de tissus et de types cellulaires (Tableau II) $[8,9]$.

\section{Les récepteurs membranaires de la protéine Gas6 et de la protéine $S$ : les récepteurs TAM}

La protéine Gas6 et la protéine $S$ sont les deux seuls ligands connus de la famille des récepteurs à activité tyrosine kinase TAM (pour Tyro3, Axl, Mer) dont les membres sont Axl (nommé également Ufo et Ark), Tyro3 (nommé également Rse, Brt, Sky) et Mer (nommé également c-Eyk) (Figure 2b) $[10,11]$. Les récepteurs TAM possèdent deux motifs immunoglobulin-like (domaine Ig) et deux motifs fibronectine de type III (domaine FNIII) extracellulaires ainsi qu'un domaine tyrosine kinase intracellulaire (Figure 2C) [11].

\section{Des phénotypes inattendus associés à l'invalidation des gènes codant pour les récepteurs TAM : les leçons apprises des souris transgéniques}

L'activation des récepteurs TAM constitue un mécanisme indispensable au bon déroulement de la réponse immunitaire (Tableau III). Les souris dont les 3 gènes codant pour les récepteurs TAM sont inactivés [12] présentent une dérégulation du système immunitaire caractérisée par de sévères désordres lympho-prolifératifs. Ces désordres pourraient s'expliquer par l'incapacité de Gas6 à exercer ses 
effets anti-apoptotiques et anti-inflammatoires via son récepteur Axl [13, 14]. En outre, les macrophages dérivés de souris $\mathrm{Mer}^{-1-}$ sont capables de lier les cellules apoptotiques mais pas de les éliminer [15].

Les souris mâles dont les trois gènes codant pour les récepteurs TAM ont été invalidés présentent une infertilité consécutive à l'obturation des tubes séminifères par des cellules apoptotiques et des corps résiduels. En effet, le processus de la spermatogenèse produit des cellules apoptotiques et des corps résiduels qui sont normalement phagocytés par les cellules de Sertoli. Cette fonction de phagocytose par les cellules de Sertoli est défectueuse chez les souris dont les trois gènes codant pour les récepteurs TAM ont été invalidés [16].

Les protéines $S$ et Gas6, tout comme leurs récepteurs TAM, sont exprimées dans le système nerveux central [17]. En outre, la $\gamma$-carboxylase est fortement exprimée dans le neuroépithélium périventriculaire du système nerveux central lors du développement et son expression persiste à l'âge adulte dans le cerveau [18]. Cela suggère que la protéine Gas6 et la protéine $S$ produites localement seraient $\gamma$-carboxylées et donc capables d'activer leurs récepteurs TAM de manière autocrine ou paracrine [18]. Les souris dont les trois gènes codant pour les récepteurs TAM ont été invalidés présentent des anomalies neurologiques caractérisées par une augmentation de la dégénérescence cellulaire et du nombre de cellules en apoptose au niveau du cervelet, de l'hippocampe et du néocortex [16] (Tableau III). Les protéines $S$ et Gas6 jouent également un rôle essentiel dans le fonctionnement normal de la rétine. En effet, des travaux in vitro ont confirmé que Gas6 et la protéine $S$ stimulent la phagocytose des segments externes des
A



Site de clivage par des sérine protéases

C

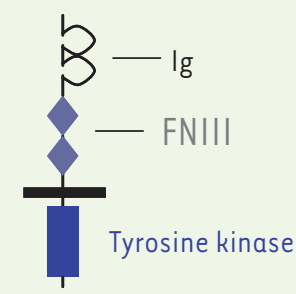

cellules cibles

Types d'organes et de cellules cibles
B

Foie, plaquettes, poumons, Rétine (épithélium pigmentaire), rein, cellules hématopoïétiques, tissus nerveux, Testicules (cellules de Sertoli et Leydig)

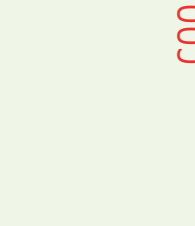


photorécepteurs par l'épithélium pigmentaire rétinien [19]. Ce phénomène appelé shedding permet le renouvellement des segments externes des photorécepteurs. Son dysfonctionnement entraîne une dégénérescence de la rétine et conduit à la cécité. Une souche de rats appelés RCS (Royal college of surgeons) présente une dégénérescence rétinienne caractérisée par un défaut de la phagocytose des segments externes des photorécepteurs (Tableau III) [20]. Les études de clonage positionnel menées chez le rat RCS ont permis de lier cette dégénérescence rétinienne à une mutation nulle du gène $\operatorname{Mer~[20].~En~}$ outre, l'invalidation du gène Mer chez la souris entraîne un phénotype dystrophique similaire à celui des rats RCS [21]. En accord avec ces observations, l'invalidation des 3 gènes codant pour les récepteurs TAM chez la souris conduit à une cécité postnatale consécutive à des dégénérescences des photorécepteurs (Tableau III) [16].

Enfin, Gas6 et la protéine S, via l'activation du récepteur Tyro3, stimulent l'activité des ostéoclastes [22] dont le rôle dans l'ostéogenèse est l'élimination de petites surfaces osseuses (résorption osseuse). Des anomalies au niveau de l'ostéogenèse n'ont pas été, pour le moment, décrites pour les souris $\mathrm{Gas}^{-/-}$ou pour les souris dont les gènes codant pour les récepteurs TAM ont été invalidés. Cependant, chez l'homme, une déficience congénitale de la protéine $S$ a été associée à une baisse de la densité minérale osseuse ainsi qu'à de l'ostéonécrose [23].

Aucun laboratoire n'a réussi pour le moment à produire des souris protéine $\mathrm{S}^{-/ \text {. }}$. Les souris $\mathrm{Gas}^{-/-}$présentent une faible incidence de thrombose (Tableau III) qui serait en partie due à un défaut d'agrégation plaquettaire $[16,24]$. Ces travaux trouvent une application thérapeutique directe dans la mesure où des inhibiteurs de Gas6 pourraient constituer un traitement efficace contre les phénomènes de thrombose.

L'ensemble des données précédemment citées montre que l'inactivation des gènes codant pour les récepteurs TAM conduit dans la majorité des cas à une dérégulation du processus de phagocytose entraînant des anomalies fonctionnelles. Il en découle que la régulation de la phagocytose serait le dénominateur commun par lequel la protéine Gas6 et la protéine $S$ réguleraient un grand nombre de processus physiologiques.

\section{L'activation des récepteurs TAM au menu du signal «mangez-moi »}

L'élimination de la cellule apoptotique comporte une phase de reconnaissance et de liaison puis une phase d'internalisation [15]. Lors du déclenchement de l'apoptose, le cytochrome C libéré par les mitochondries dans le cytosol, catalyse l'oxydation des phosphatidylsérines, entraînant leur translocation à la surface externe de la membrane plasmique [25] (Figure 3). La localisation des résidus phosphatidylsérines oxydés à la surface externe de la membrane plasmique constitue un signal de reconnaissance des cellules apoptotiques par les phagocytes appelé aussi signal «mangez-moi». Un récepteur spécifique des phosphatidylsérines oxydées a récemment

\section{Souris transgéniques}

\begin{tabular}{|c|c|c|c|c|c|c|}
\hline $\begin{array}{l}\text { Gène } \\
\text { invalidé }\end{array}$ & Gas6 & Axl & Tyro3 & Mer & $\begin{array}{l}\text { Axl/Tyro3/Mer } \\
\text { (triple KO) }\end{array}$ & Mer \\
\hline $\begin{array}{l}\text { Système } \\
\text { nerveux }\end{array}$ & $\begin{array}{l}\text { Non } \\
\text { exploré }\end{array}$ & $\begin{array}{l}\text { Non } \\
\text { exploré }\end{array}$ & $\begin{array}{l}\text { Non } \\
\text { exploré }\end{array}$ & $\begin{array}{l}\text { Non } \\
\text { exploré }\end{array}$ & -Anomalies neurologiques [16] & Non exploré \\
\hline $\begin{array}{l}\text { Système } \\
\text { immunitaire }\end{array}$ & $\begin{array}{l}\text { - Protection } \\
\text { contre des } \\
\text { lésions réna- } \\
\text { les induites } \\
\text { [47] }\end{array}$ & \multicolumn{2}{|c|}{$\begin{array}{l}\text { - Élévation du taux } \\
\text { d'autoanticorps } \\
{[12,16] \text {. }}\end{array}$} & $\begin{array}{l}\text { Défaut de phagocytose des } \\
\text { cellules apoptotiques par les } \\
\text { macrophages et les monocytes } \\
\text { [12] }\end{array}$ & $\begin{array}{l}\text { - Désordres auto-immuns [12]: } \\
\text { - Augmentation de l'apoptose. } \\
\text { - Thromboses et hémorragies dans } \\
\text { divers tissus. }\end{array}$ & Non exploré \\
\hline $\begin{array}{l}\text { Coagulation, } \\
\text { Agrégation } \\
\text { plaquettaire }\end{array}$ & \multicolumn{4}{|c|}{ - Défaut d'agrégation plaquettaire [48] } & Non exploré & Non exploré \\
\hline $\begin{array}{l}\text { Système } \\
\text { visuel }\end{array}$ & $\begin{array}{l}\text { - Rétine } \\
\text { normale [19] }\end{array}$ & $\begin{array}{l}\text { Non } \\
\text { exploré }\end{array}$ & $\begin{array}{l}\text { Non } \\
\text { exploré }\end{array}$ & - Défaut de la phagocytose des & 1otorécepteurs, dégénérescence rétin & enne $[16,20,21]$ \\
\hline
\end{tabular}

Tableau III. Phénotypes associés à l'invalidation génique des récepteurs TAM chez la souris et à la mutation nulle du récepteur Mer chez le rat RCS. Mer: mutation héréditaire chez les rats RCS. 
été identifié dans les macrophages [26]. Les principales opsonines connues des phosphatidylsérines oxydées sont la protéine MFG-\&8 (milk fat globule $\varepsilon G F$ factor 8 ) dont les récepteurs sont les intégrines $\alpha v \beta 3 / \alpha v \beta 5$ et la protéine Gas 6 dont les récepteurs sont les TAM. En effet, l'inhibition de l'interaction de MFG-E8 ou de Gas6 avec leurs récepteurs respectifs résulte en un défaut d'élimination de cellules apoptotiques [27]. La protéine Gas6 interagit avec les phosphatidylsérines oxydées par son domaine Gla et avec ses récepteurs TAM, présents à la surface du macrophage, par son domaine SHBG [28]. Cela favoriserait l'interaction entre la cellule apoptotique et le phagocyte [29] (Figure 3). Par ailleurs, le fractionnement du sérum a révélé que la protéine $S$ est le principal facteur sérique capable de lier les phosphatidylsérines et de stimuler la phagocytose des cellules lymphoïdes en apoptose par les macrophages [30]. À ce titre, les protéines $S$ et Gas 6 se comportent comme de vraies jumelles.

La cascade de signalisation Rho-GTPase Racl régule la polymérisation des filaments d'actine permettant un changement de la morphologie du macrophage et l'internalisation de la cellule apoptotique. En réponse à l'activation des intégrines par leur ligand MFG-£8, les protéines cytosoliques Crk, DOCK180 et ELMO forment un complexe trimérique sous-membranaire capable d'activer Racl[31] (Figure 3). Le complexe ELMO-DOCK180 ainsi que la protéine Vavl constituent le facteur d'échange (GEF) de Racl et participent à son activation [32]. L'activation du récepteur Mer par Gas6 conduit au recrutement de la PI3K et de la phospholipase $C \gamma$, et à la phosphorylation de Vavl, activant de ce fait la cascade de signalisation Rho-GTPase Racl et favorisant ainsi la phagocytose de cellules apoptotiques [33].

\section{Perspectives}

Jusqu'en 1993, la protéine Gas6 était inconnue et la fonction de la protéine $S$ était limitée à l'inhibition de la coagulation du sang. Les récepteurs TAM ont été identifiés comme étant des récepteurs à activité tyrosine kinase sans ligands connus et nommés récepteurs orphelins [34]. Plus tard, Gas6 a été découverte à la

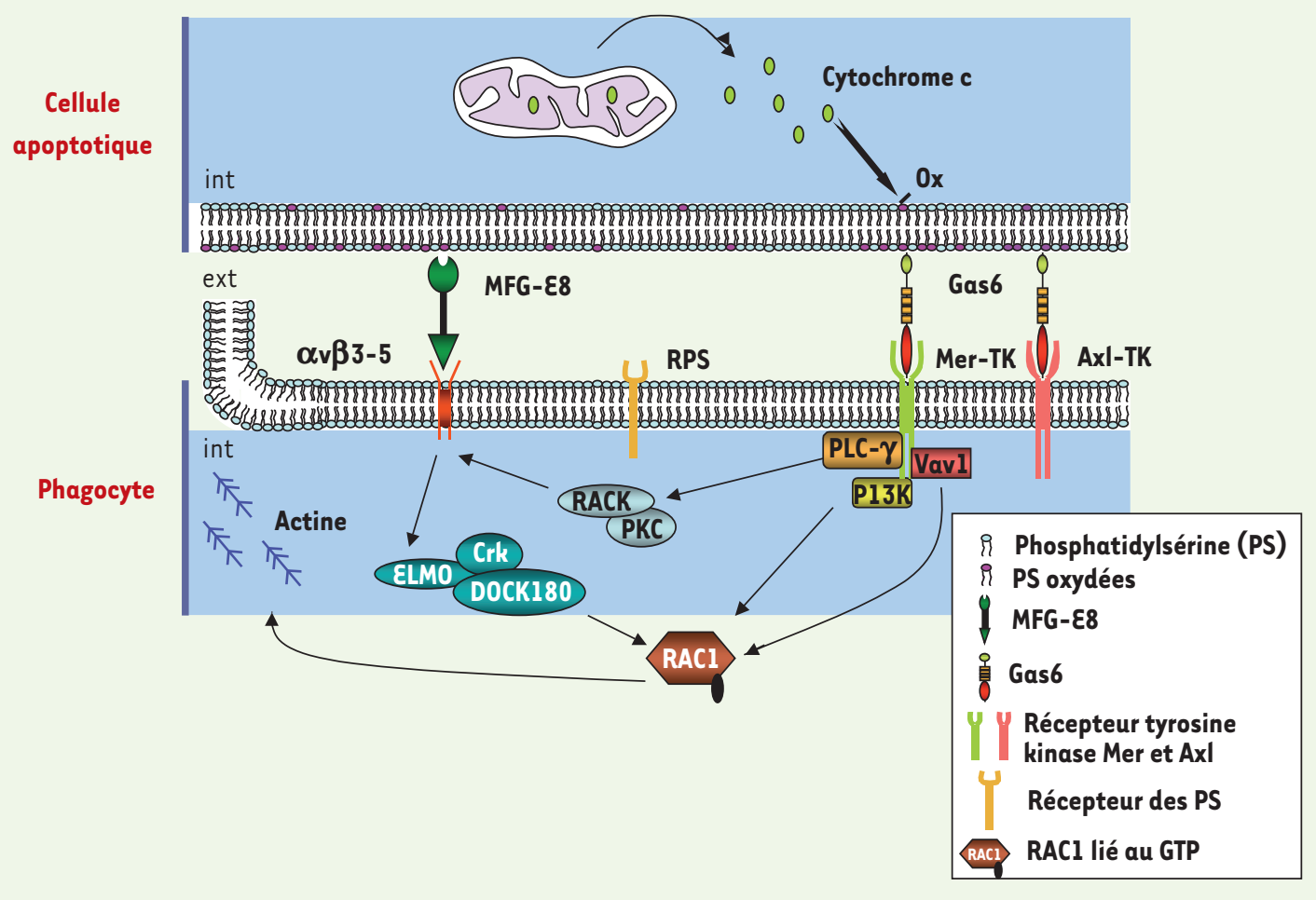

Figure 3. Implication des récepteurs TAM dans la reconnaissance et la phagocytose des cellules apoptotiques par le macrophage. La signalisation Gas6/Axl serait essentiellement impliquée dans l'interaction cellule/cellule et l'adhérence cellulaire. La signalisation Gas6/Mer est la voie majeure impliquée dans la phagocytose. La liaison de Gas6 à son récepteur Mer active la kinase phosphatidylinositol 3'-OH Kinase (PI3K). Cette voie est impliquée dans l'activation de RACl et de la phospholipase $C \gamma(P L C-\gamma)$ et module ainsi la signalisation par les intégrines $\alpha v \beta 3-5$ via le complexe RACK/PKC. L'activation du récepteur Mer conduit également à la phosphorylation du facteur d'échange Vavl. Le remodelage du cytosquelette d'actine nécessaire à l'internalisation de la cellule apoptotique par le phagocyte est médié par les intégrines qui stimulent la formation du complexe Crk/ELMO/DOCK180 responsable de l'activation de Racl. 
fois en tant que nouveau membre de la famille des protéines vitamine K-dépendantes, comme un homologue de la protéine $S$ et en tant que le ligand (avec la protéine S) des récepteurs TAM [34]. Depuis, l'étude des processus cellulaires activés par les récepteurs TAM, ainsi que des phénotypes associés à l'invalidation des gènes codant pour les récepteurs TAM ont ouvert un nouvel horizon de recherche qui fascine par sa complexité, son étendue et par ses nombreuses applications thérapeutiques potentielles. L'apoptose a occupé les devants de la scène depuis plus d'une décennie. Elle semble céder progressivement la place à l'étude des phénomènes post-apoptotiques et notamment la phagocytose dont la régulation serait le dénominateur commun à la croisée des voies de signalisation des récepteurs TAM. Enfin, la $\gamma$-carboxylation étant liée à l'activité biologique des protéines $S$ et Gas6, l'impact des traitements AVK sur les fonctions nouvellement découvertes des protéines vitamine K-dépendantes devrait faire l'objet d'études cliniques. Cela permettrait de prévenir certains effets secondaires de la warfarine, comme par exemple le cas récemment décrit d'un patient déficitaire en protéine $S$ soumis à un traitement AVK chronique et présentant une hypotrophie testiculaire [35]. $\diamond$

\section{SUMMARY}

Gas-6 and protein S:

vitamin K-dependent factors and ligands

for the TAM tyrosine kinase receptors family

The $\gamma$-carboxyglutamate-containing proteins are a family of secreted vitamin K-dependent proteins in which some glutamyl residues are post-translationally modified to $\gamma$-carboxyglutamic acid residues. A vitamin K-dependent $\gamma$-glutamyl carboxylase enzyme catalyses this post-translational modification. The $\gamma$-carboxylase reaction requires vitamin $K$ in its reduced form, vitamin $K$ hydroquinone, and generates $\gamma$-carboxyglutamate and vitamin $K 2,3$,-epoxide which is then recycled back to the hydroquinone form by a vitamin $K$ reductase system. Warfarin blocks the vitamin $K$ cycle and hence inhibits the $\gamma$-carboxylase reaction, and this property of Warfarin has led to its wide use in anticoagulant therapy. Until recently, interest in vitamin K-dependent proteins was mostly restricted to the field of hematology. However, the discovery that the anti-coagulant factor protein $S$ and its structural homologue Gas6 (growth arrest-specific gene 6), two vitamine K-dependent proteins, are ligands for the Tyro3/Axl/Mer family of related tyrosine kinase receptors has opened up a new area of research. Moreover, the phenotypes associated with the invalidation of genes encoding vitamin K-dependent proteins or their receptors revealed their implication in regulating phagocytosis during many cell differentiation phenomena such as retinogenesis, neurogenesis, osteogenesis, and spermatogenesis. Additionally, protein $\mathrm{S}$ was identified as the major factor responsible for serum-stimulated phagocytosis of apoptotic cells. Therefore, the elucidation of the molecular mechanisms underlying the role of vitamin $\mathrm{K}$-dependent proteins in regulating apoptotic cell phagocytosis may lead to a better understanding of the physiopathology of cell differentiation and could form the framework of new therapeutic strategies aiming at a selective targeting of cell phagocytosis associated pathologies. $\diamond$

\section{REMERCIEMENTS}

Nous remercions très vivement tous les collègues qui ont eu l'amabilité de relire cet article. Le travail de notre groupe «Fonctions des protéines $\gamma$-carboxylées dans la différenciation cellulaire » a reçu le soutien financier de la Ligue Nationale Contre le Cancer-Poitou-Charentes et de RetinaFrance. AG bénéfice d'une allocation de recherche du Ministère de la Recherche et de la Technologie.

\section{RÉFÉRENCES}

1. Mann KG, Nesheim ME, Church WR, et al. Surface-dependent reactions of the vitamin K-dependent enzyme complexes. Blood $1990 ; 76$ : 1-16.

2. Wallin R, Hutson SM. Warfarin and the vitamin K-dependent gammacarboxylation system. Trends Mol Med $2004 ; 10$ : 299-302.

3. Furie B, Bouchard BA, Furie BC. Vitamin K-dependent biosynthesis of gamma-carboxyglutamic acid. Blood $1999 ; 93: 1798-808$.

4. Scully M. Warfarin therapy. The Biochemist $2002 ; 24: 15-7$.

5. Preissner KT, Nawroth PP, Kanse SM. Vascular protease receptors : integrating haemostasis and endothelial cell functions. J Pathol 2000; $190: 360-72$.

6. Carmeliet P. Biomedicine. Clotting factors build blood vessels. Science $2001 ; 293: 1602-4$

7. Leger AJ, Covic L, Kuliopulos A. Protease-activated receptors in cardiovascular diseases. Circulation 2006; 114 : 1070-7.

8. Manfioletti G, Brancolini C, Avanzi G, Schneider C. The protein encoded by a growth arrest-specific gene (gas6) is a new member of the vitamin $\mathrm{K}$-dependent proteins related to protein $\mathrm{S}$, a negative coregulator in the blood coagulation cascade. Mol Cell Biol 1993; 13 : 4976-85.

9. He X, Shen L, Bjartell A, Dahlback B. The gene encoding vitamin Kdependent anticoagulant protein $S$ is expressed in multiple rabbit organs as demonstrated by northern blotting, in situ hybridization, and immunohistochemistry. J Histochem Cytochem 1995 ; 43 : 85-96.

10. Prasad D, Rothlin CV, Burrola P, et al. TAM receptor function in the retinal pigment epithelium. Mol Cell Neurosci 2006 ; 33: 96-108.

11. Hafizi S, Dahlback B. Signalling and functional diversity within the AxI subfamily of receptor tyrosine kinases. Cytokine Growth Factor Rev 2006 ; $17: 295-304$.

12. Lu Q, Lemke $G$. Homeostatic regulation of the immune system by receptor tyrosine kinases of the Tyro 3 family. Science 2001 ; 293 : 306-11.

13. Demarchi F, Verardo R, Varnum B, et al. Gas6 anti-apoptotic signaling requires NF-kappa B activation. J Biol Chem 2001 ; 276 : 31738-44.

14. Sharif MN, Sosic D, Rothlin CV, et al. Twist mediates suppression of inflammation by type I IFNs and AxI. J Exp Med 2006; 203 : 1891-901.

15. Scott RS, McMahon EJ, Pop SM, et al. Phagocytosis and clearance of apoptotic cells is mediated by MER. Nature $2001 ; 411: 207-11$.

16. Lu $Q$, Gore $M$, Zhang $Q$, et al. Tyro- 3 family receptors are essential regulators of mammalian spermatogenesis. Nature 1999; $398: 723-8$.

17. Prieto AL, Weber JL, Lai C. Expression of the receptor protein-tyrosine kinases Tyro-3, Axl, and mer in the developing rat central nervous system. J Comp Neurol $2000 ; 425$ : 295-314.

18. Romero $\varepsilon \varepsilon$, Velazquez-Estades LJ, Deo R et al. Cloning of rat vitamin Kdependent gamma-glutamyl carboxylase and developmentally regulated gene expression in postimplantation embryos. Exp Cell Res 1998; $243: 334-46$.

19. Hall MO, Obin MS, Heeb MJ, et al. Both protein S and Gas6 stimulate outer segment phagocytosis by cultured rat retinal pigment epithelial cells. Exp Eye Res 2005 ; 81 : 581-91.

20. D'Cruz PM, Yasumura D, Weir J, et al. Mutation of the receptor tyrosine kinase gene Mertk in the retinal dystrophic RCS rat. Hum Mol Genet 2000 ; $9: 645-51$.

21. Duncan JL, LaVail MM, Yasumura D, et al. An RCS-like retinal dystrophy phenotype in mer knockout mice. Invest Ophthalmol Vis Sci 2003; 44: 826-38.

22. Katagiri M, Hakeda $Y$, Chikazu D, et al. Mechanism of stimulation of osteoclastic bone resorption through Gas6/Tyro 3, a receptor tyrosine kinase signaling, in mouse osteoclasts. J Biol Chem 2001; 276 : 7376-82.

23. Pierre-Jacques $\mathrm{H}$, Glueck CJ, Mont MA, et al. Familial heterozygous protein-S deficiency in a patient who had multifocal osteonecrosis. A case report. J Bone Joint Surg Am 1997 ; 79 : 1079-84. 
24. Angelillo-Scherrer A, Burnier L, Flores N, et al. Role of Gas6 receptors in platelet signaling during thrombus stabilization and implications for antithrombotic therapy. J Clin Invest $2005 ; 115: 237-$ 46.

25. Jiang J, Kini V, Belikova N, et al. Cytochrome $\mathrm{c}$ release is required for phosphatidylserine peroxidation during Fas-triggered apoptosis in lung epithelial A549 cells. Lipids 2004 ; 39 : 1133-42.

26. Fadok VA, Bratton DL, Rose DM, et al. A receptor for phosphatidylserine-specific clearance of apoptotic cells. Nature $2000 ; 405: 85-90$.

27. Cohen PL, Caricchio R, Abraham V, et al. Delayed apoptotic cell clearance and lupus-like autoimmunity in mice lacking the c-mer membrane tyrosine kinase. J Exp Med 2002; 196 : $135-40$.

28. Nakano $\mathrm{T}$, Ishimoto $\mathrm{Y}$, Kishino J, et al. Cell adhesion to phosphatidylserine mediated by a product of growth arrest-specific gene 6. J Biol Chem 1997 ; 272 : 29411-4.

29. Neubauer A, Fiebeler A, Graham DK, et al. Expression of axl, a transforming receptor tyrosine kinase, in normal and malignant hematopoiesis. Blood $1994 ; 84: 1931-41$.

30. Anderson HA, Maylock CA, Williams JA, et al. Serum-derived protein S binds to phosphatidylserine and stimulates the phagocytosis of apoptotic cells. Nat Immunol 2003; $4: 87-91$.

31. Wu Y, Tibrewal N, Birge RB. Phosphatidylserine recognition by phagocytes : a view to a kill. Trends Cell Biol $2006 ; 16: 189-97$.

32. Brugnera $\varepsilon$, Haney L, Grimsley C, et al. Unconventional Rac-GEF activity is mediated through the Dock180-ELM0 complex. Nat Cell Biol 2002 ; $4: 574-82$.

33. Todt JC, Hu B, Curtis JL. The receptor tyrosine kinase MerTK activates phospholipase C gamma2 during recognition of apoptotic thymocytes by murine macrophages. J Leukoc Biol 2004 ; 75 : 705-13.

34. Stitt TN, Conn G, Gore M, et al. The anticoagulation factor protein S and its relative, Gas6, are ligands for the Tyro 3/Axl family of receptor tyrosine kinases. Cell 1995; $80: 661-70$.

35. Mckay D, Marron C, Brown R. Testicular infarction secondary to protein $S$ deficiency : a case report. BMC Urol $2006 ; 6: 17$.

36. Feistritzer C, Lenta R, Riewald M. Protease-activated receptors-1 and -2 can mediate endothelial barrier protection : role in factor Xa signaling. J Thromb Haemost 2005 ; 3: 2798-805.

37. Yang X, Walsh PN. An ordered sequential mechanism for factor IX and factor IXa binding to platelet receptors in the assembly of the factor X-activating complex. Biochem / 2005 ; $390: 157-67$.

38. Guo H, Liu D, Gelbard H, et al. Activated protein C prevents neuronal apoptosis via protease activated receptors 1 and 3 . Neuron $2004 ; 41: 563-72$

39. Domotor $\varepsilon$, Benzakour 0 , Griffin JH, et al. Activated protein C alters cytosolic calcium flux in human brain endothelium via binding to endothelial protein $C$ receptor and activation of protease activated receptor-1. Blood 2003; $101: 4797-801$.
40. Broze GJ Jr. Protein Z-dependent regulation of coagulation. Thromb Haemost $2001 ; 86: 8-13$.

41. Hafizi S, Dahlback B. Gas6 and protein S. Vitamin K-dependent ligands for the Axl receptor tyrosine kinase subfamily. FEBS J 2006 ; 273 : 523144.

42. Maillard C, Berruyer M, Serre CM, et al. Protein-S, a vitamin K-dependent protein, is a bone matrix component synthesized and secreted by osteoblasts. Endocrinology $1992 ; 130$ : 1599-604.

43. Murshed M, Schinke T, McKee MD, Karsenty G. Extracellular matrix mineralization is regulated locally; different roles of two gla-containing proteins. J Cell Biol $2004 ; 165: 625-30$.

44. Ducy P, Desbois C, Boyce B, et al. Increased bone formation in osteocalcin-deficient mice. Nature 1996 ; 382: 448-52.

45. Kulman JD, Harris JE, Xie L, Davie EW. Identification of two novel transmembrane gamma-carboxyglutamic acid proteins expressed broadly in fetal and adult tissues. Proc Natl Acad Sci USA 2001 ; 98: 1370-5.

46. Kulman JD, Harris JE, Haldeman BA, Davie EW. Primary structure and tissue distribution of two novel proline-rich gamma-carboxyglutamic acid proteins. Proc Natl Acad Sci USA 1997 ; 94 : 9058-62.

47. Yanagita $M$, Ishimoto $Y$, Arai $H$, et al. Essential role of Gas 6 for glomerular injury in nephrotoxic nephritis. J Clin Invest 2002; 110 : 239-46.

48. Angelillo-Scherrer A, de Frutos P, Aparicio C, et al. Deficiency or inhibition of Gas6 causes platelet dysfunction and protects mice against thrombosis. Nat Med $2001 ; 7: 215-21$.



ISBN : 978-2-8425-4118-7 96 pages

\section{Bon de commande}

À retourner à EDK, 2, rue Troyon - 92316 Sèvres Cedex

Tél. : 0155641393 - Fax : 0155641394 - E-mail : edk@edk.fr

NOM :

Prénom :

Adresse :

Code postal :

Ville :

Pays :

Fonction :

Je souhaite recevoir l'ouvrage $13^{\mathrm{e}}$ Symposium Recherche et Glaucome :

$25 €+3 €$ de port $=\mathbf{2 8} € \mathbf{T T C}$

en .................. exemplaire, soit un total de .............................................. €

$\square$ Par chèque, à l'ordre de $\mathbf{E} \mathbf{D}$ K

$\square$ Par carte bancaire : $\square$ Visa $\square$ Eurocard/Mastercard

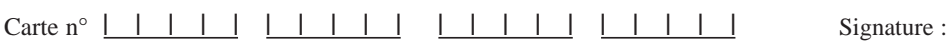

Date d'expiration : $\quad \leq 1|1| 1$

$\mathrm{N}^{\circ}$ de contrôle au dos de la carte :
TIRÉS À PART

0. Benzakour

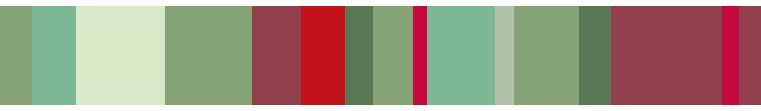

